\title{
Characterization of the Pharmaceutical Effect of Drugs on Atherosclerotic Lesions in Vivo Using Integrated Fluorescence Imaging and Raman Spectral Measurements
}

\author{
Yi-Cyun Yang, ${ }^{\dagger}$ Wei-Tien Chang, ${ }^{\ddagger}$ Shao-Kang Huang, ${ }^{\dagger}$ and Ian Liau ${ }^{*} \dagger$ \\ ${ }^{\dagger}$ Department of Applied Chemistry and Institute of Molecular Science, National Chiao Tung University, Hsinchu 300, Taiwan \\ ${ }^{\ddagger}$ National Taiwan University Hospital and College of Medicine, Taipei 100, Taiwan
}

\begin{abstract}
Direct assessment of the vascular lesions of model animals in vivo is important for the development of new antiatherosclerotic drugs. Nevertheless, biochemical analysis of the lipid profile in blood in vitro remains the most common way to evaluate the therapeutic effect of drugs targeting atherosclerosis because of an inherent difficulty to access the vascular wall. Using hypercholesterolemic zebrafish, we present an orchestrated application of Raman spectral measurements and confocal fluorescence imaging to interrogate the pharmacological response of atherosclerotic lesions in situ and in vivo. For demonstration, we investigated two commonly prescribed antihyperlipidemic drugs, ezetimibe and atorvastatin. The treatment of ezetimibe or atorvastatin alone decreased effectively the deposition of lipids in the vascular wall, and a combined dose showed a synergistic effect. Atorvastatin exerted a profound antioxidative effect on vascular fatty lesions. Analysis of individual lesions shows further that these lesions exhibited a heterogeneous response to the treatment of atorvastatin; a significant fraction of, but not all, the lesions became nonoxidized after the intervention. Beyond its efficacies in suppressing both the accumulation and oxidation of vascular lipids, atorvastatin expedited the clearance of vascular lipids. The possession of pleotropic (multiple) therapeutic effects on vascular fatty lesions of hypercholesterolemic zebrafish by atorvastatin is notably consistent with the known pharmaceutical effects of this drug on human beings. These results improve our understanding of the antiatherosclerotic effect of drugs. We envisage that our approach has the potential to become a platform to predict the pharmaceutical effects of new drugs aiming to cure human atherosclerotic diseases.
\end{abstract}

\begin{abstract}
A therosclerotic cardiovascular disease is a leading cause of death. ${ }^{1,2}$ In particular, an accumulation of low-density lipoprotein $(\mathrm{LDL})$ in the vascular wall and its oxidative modification are two vital events that underline the pathogenesis of atherosclerosis. ${ }^{2-4}$ Specifically, oxidatively modified LDL (oxLDL) acts as a chemoattractant to induce an extravasation of monocytes; these monocytes subsequently differentiate into macrophages and engulf the infiltrated LDL in the vascular wall, eventually leading to a formation of the lipid core of atheroma. ${ }^{3,5}$ As a result, suppression of the vascular accumulation of LDL and its oxidative modification have been long regarded as major therapeutic targets to prevent and to treat atherosclerosis. ${ }^{2,6}$

Pharmacological development requires inevitably an assessment of the therapeutic effects of drugs on animal models of human diseases. Among model organisms commonly employed for drug screening, the zebrafish (Danio rerio) is particularly attractive because of its possession of numerous unique features such as rapid growth, ease of administration of drugs, and semitranslucency. Miller and co-workers showed that zebrafish fed with high-cholesterol diets (HCD) developed crucial pathological features of atherosclerosis in the early stage. ${ }^{7}$ They demonstrated determination of oxLDL in the vascular wall using a novel transgenic fish line that expresses IK17 (antibody of MDA-LDL) ${ }^{8}$ and suggested the utility of zebrafish
\end{abstract}

to screen drugs with antiatherosclerotic effects. ${ }^{9}$ Despite the unprecedented advance, limitations associated with their approach remain. First, the quantity of antibodies expressed among individual fish may not be homogeneous, which might prejudice accurate determination of oxLDL. Second, because the antibody labeled only oxLDL, unmodified LDL is presumably invisible with this approach, so that information about the extent of oxidation of vascular fatty lesions hence remains incomplete. Furthermore, certain questions central to justify the use of zebrafish to predict drugs of therapeutic effects remain largely unanswered; for instance, whether drugs of known antiatherosclerotic effects on human beings exert similar therapeutic effects on zebrafish is not yet critically examined.

Raman spectral analysis can reveal the molecular "fingerprints" of matter without labeling ${ }^{10-13}$ and has been extensively employed to characterize cells and biological tissues, ${ }^{14-18}$ integration of which with optical imaging further enables a spectral assessment of subcellular organelles at the level of single cells. ${ }^{19-23}$ Despite an enabling technology, application of Raman spectral measurements to investigate pharmaceutical effects on zebrafish models of human diseases has not been

Received: December 15, 2013

Accepted: March 15, 2014

Published: March 16, 2014 
reported yet. Herein, we demonstrate an orchestrated application of confocal fluorescence imaging and Raman spectral measurements to assess the therapeutic response of hypercholesterolemic zebrafish in vivo. Our approach is unique with regard to either biochemical analysis of plasma lipids or determination of total body fat; the former provides information only indirectly related to the pathological abnormalities of vascular fatty lesions, whereas the latter contains not only the vascular lipids but also the fat stored in subcutis and organs or the LDL circulating in blood. We investigated two commonly prescribed drugs (ezetimibe and atorvastatin) that have been demonstrated to have potent antihyperlipidemic efficacies through distinct mechanisms. We show that atorvastatin possesses profound pleotropic therapeutic effects on atherosclerotic lesions, beyond its efficacies in suppressing the accumulation of lipids in the vascular wall. We expect that our work could not only improve our understanding of the pharmacological activities of antiatherosclerotic drugs but also facilitate the development of drugs targeting atherosclerotic diseases.

\section{MATERIALS AND METHODS}

The Animal Investigation Committee of National Chiao Tung University approved this work (permit No. 00990001).

Reagents. Cholesterol, diethyl ether, fluorescein isothiocyanate (FITC)-dextran ( $\mathrm{MM}=150 \mathrm{kDa})$, tricaine, isoflurane, copper(II) sulfate pentahydrate, low-density lipoprotein (LDL) from human plasma and atorvastatin (Sigma Aldrich), artificial artemia (Azoo), cholesteryl BODIPY- $\mathrm{C}_{11}$ (fluorescent analogue of cholesterol, Invitrogen), and ezetimibe (Santa Cruz Biotechnology) were acquired from the indicated sources.

Handling of Zebrafish. Breeding colonies of $\mathrm{AB}$ strain zebrafish (Danio rerio), provided by Taiwan Zebrafish Core Facility, were kept in small aquaria according to protocols described elsewhere. ${ }^{24}$ Hypercholesterolemic zebrafish were induced with diets enriched with cholesterol according to protocols modified from the literature. ${ }^{7}$ The high-cholesterol diet (HCD) was prepared on mixing cholesterol with artificial artemia ( $1 \mathrm{~g}, 10 \%$ mass $/$ mass $)$ in diethyl ether $(3 \mathrm{~mL})$ and then finely ground after evaporation of the solvent. The normal diet was prepared with artificial artemia without addition of cholesterol. To facilitate imaging of the deposited lipids in the vascular wall, both diets were replenished with the fluorescent analogue of cholesterol $(10 \mu \mathrm{g} / \mathrm{g})$ in a manner similar to that used to prepare HCD. ${ }^{7}$ Beginning from five days post fertilization ( $\mathrm{dpf})$, the larvae were fed once a day for the designated durations. To administer the intervention, we supplemented the diet with the drug in the designed dose, and the supplemented diet was fed to the fish in a manner similar to the diet that contained no drug. Before imaging or spectral measurements, a zebrafish larva was anesthetized with a solution containing a mixture of tricaine $(100 \mathrm{ppm})$ and isoflurane $(100 \mathrm{ppm}){ }^{25}$ To facilitate visualization of the vascular lumen, the anesthetized larva was injected with FITC-dextran.

Confocal Fluorescence Imaging and Raman Spectral Measurements. Fluorescence imaging and Raman spectral measurements were performed with a system (Figure 1) modified from that reported. ${ }^{19,20}$

For fluorescence imaging, a blue laser (488 nm, Newport) was employed to excite FITC-dextran (vascular lumen) and an orange laser (561 nm, Cobolt) for cholesteryl BODIPY (fatty lesions). Throughout the experiments, the powers were kept at

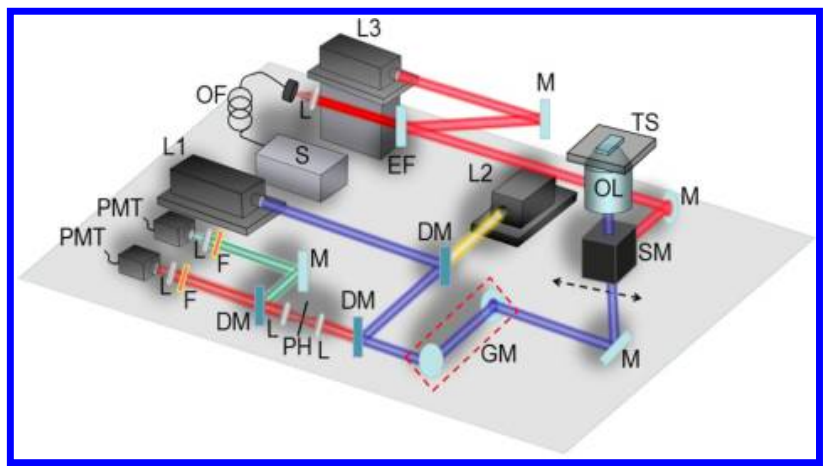

Figure 1. Schematic of the experimental setup for integrated Raman spectral measurements and confocal fluorescence imaging. L1-L3: laser $(\lambda=488,561$, and $632.8 \mathrm{~nm})$; M: mirror; EF: edge filter; OF: optical fiber; S: spectrometer; TS: translation stage; OL: objective lens; SM: switchable mirror; GM: galvanometric mirrors; DM: dichroic mirror; L: lens; PH: pinhole; F: emission filter; PMT: photomultiplier tube.

$200 \mu \mathrm{W}$ for the blue laser and at $2 \mathrm{~mW}$ for the orange laser, measured before entry into the confocal scanner. The signal was detected between 495 and $550 \mathrm{~nm}$ corresponding to the emission of FITC-dextran and beyond $570 \mathrm{~nm}$ for cholesteryl BODIPY. All images $(1024 \times 1024$ pixels, $10 \mathrm{~s}$ per frame $)$ were obtained with an objective lens (UPLSAPO 20×, Olympus) and rendered either with a single frame or with frames in a 40 $\mu \mathrm{m}$ stack accumulated with an interval of $2 \mu \mathrm{m}$. Threedimensional images were constructed from a stack of images with software (Imaris, Bitplane).

For Raman spectral measurements, a red $\mathrm{HeNe}$ laser $(632.8$ $\mathrm{nm}$, JDS Uniphase) was directed to the optical microscope and focused with an objective lens (UPLSAPO 60XW, Olympus). The powers, measured at the back port of the microscope, were set at $13.0 \mathrm{~mW}$ for the measurements of zebrafish in vivo and $3.8 \mathrm{~mW}$ for LDL in vitro. The region of measurements was identified from fluorescent images and was positioned to the laser focus with a translation stage that had been integrated with the microscope. The Raman signal was collected in the epi-direction and analyzed with a spectrograph (Spectra Pro2300i, Princeton Instruments). All Raman spectra were rendered on averaging four spectra obtained at the same region with integration for $10 \mathrm{~s}$ for each; the background was subtracted after the specified data processing.

Image Analysis. To determine the relative quantity of vascular lipids, we first overlaid 20 images obtained at varied depth (with an interval of $2 \mu \mathrm{m}$ ) and identified individual vascular lesions in the overlaid image as the region of interest (ROI). The intensity of the ROI were then integrated with freeware (ImageJ 1.43j, National Institutes of Health, U.S.A.). The background was determined from a region of the same area with the ROI, randomly selected within the vascular lumen. The data was reported as the intensity after subtracting the background.

Statistics. Data are represented as mean \pm SD. Comparison between two groups was made with the two-tailed Student's $t$ test. The levels of statistical significance were set at $* P<0.05$, $* * P<0.01$, and $* * * P<0.001$, respectively.

\section{RESULTS}

Characterization of Zebrafish Fed with HCD with Confocal Fluorescence Imaging and Raman Spectral Measurements. We first characterized zebrafish with confocal 


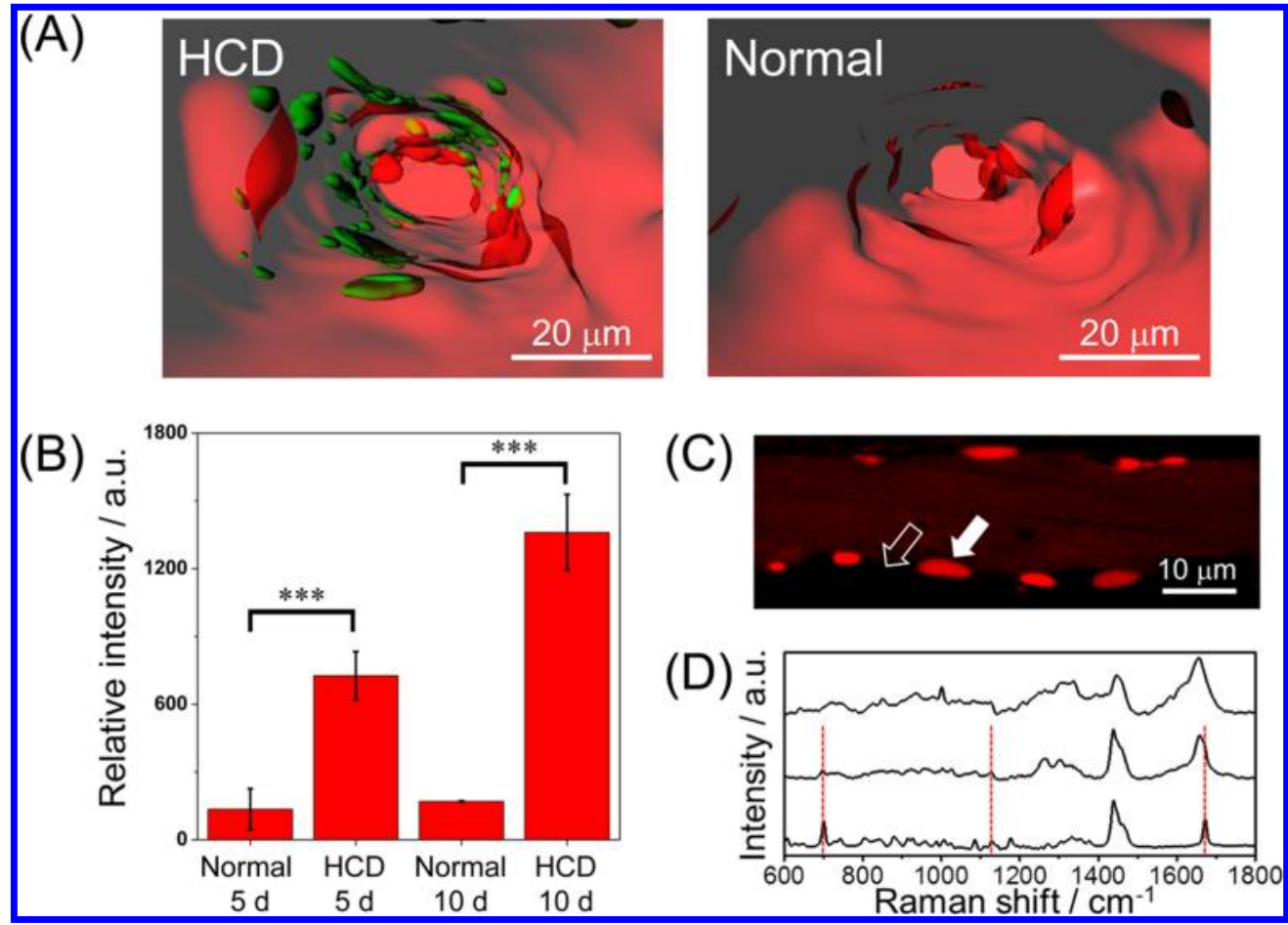

Figure 2. Imaging and Raman spectral characterization of hypercholesterolemic zebrafish. (A) Luminal view of the blood vessel of zebrafish fed with HCD or normal diets for $10 \mathrm{~d}$ (red: vascular wall; green: deposited lipids). (B) Quantification of the vascular lipids of zebrafish fed with normal or high-cholesterol diets of varied duration. (C) Representative confocal fluorescence image obtained from the vascular wall of hypercholesterolemic zebrafish. The empty and solid arrows indicate a region of no evident lesion and a fatty lesion in the vascular wall. (D) Raman spectra measured from a region of no vascular lesion (upper panel; empty arrow in C), a vascular fatty lesion (middle panel; solid arrow in C), and pure cholesterol (lower panel). Red dashed lines: characteristic Raman spectral lines of cholesterol.

fluorescence imaging. A luminal view of the blood vessel shows clearly that numerous fatty lesions (green) deposited in the blood vessel (red) of zebrafish fed with HCD for $10 \mathrm{~d}$, and these lesions located near the surface of the vascular wall (left panel, Figure 2A). In contrast, zebrafish fed with normal diets for the same duration showed sparse lipid depositions in the vascular wall (right panel, Figure 2A).

With image analysis, we quantified the lipids accumulated in the vascular wall. Our result shows that the lipid deposited in the HCD-fed zebrafish was significantly greater than that in the control fed with normal diets for the same duration; the accumulation of the vascular lipids increased progressively with the duration of feeding of HCD $(726 \pm 107$ vs $135 \pm 91$ and $1360 \pm 168$ vs $170 \pm 2$, for fish fed with HCD and normal diets for 5 and $10 \mathrm{~d}$, respectively) (Figure 2B).

Guided with fluorescence images, we performed Raman spectral measurements on individual vascular lesions and nearby regions of the vascular walls of zebrafish. We tested a larval fed with HCD for $10 \mathrm{~d}$. The Raman spectra obtained from vascular regions of no evident lesion (empty arrow, Figure 2C) consistently exhibit one sharp spectral line $\left(1003 \mathrm{~cm}^{-1}\right)$ corresponding to phenylalanine and two broad lines (12001300 and $1500-1700 \mathrm{~cm}^{-1}$ ) consistent with amide III and I, respectively (upper panel, Figure 2D). ${ }^{26-28}$ Although we cannot exclude the contribution of other sources to these spectral lines, the result indicates that protein is the dominant constituent in this region, a deduction that conforms to the structural characteristics of a healthy vascular wall. ${ }^{2}$ In comparison, the representative Raman spectrum of vascular lesions (solid arrow, Figure 2C) possesses two distinctive spectral lines at 700 and $1130 \mathrm{~cm}^{-1}$ and an embedded line at
$1670 \mathrm{~cm}^{-1}$ appearing as a shoulder on a broad spectral line (middle panel, Figure 2D). These spectral lines are consistent with the Raman characteristics of cholesterol (lower panel, Figure 2D); the result conforms to our knowledge of LDL whose fatty component comprised mainly cholesterol. ${ }^{2}$

These collective results show that zebrafish fed with HCD exhibit characteristic pathological features consistent with those of human atherosclerosis. ${ }^{2}$ More importantly, the preceding results indicate that, facilitated with the translucent nature of zebrafish, our integrated confocal fluorescence imaging and Raman spectral measurements is able to determine not only the quantity of vascular lipids but also their chemical structures noninvasively in vivo.

The ability to interrogate the vascular wall of living zebrafish with confocal fluorescence imaging and Raman spectral measurements arouses an unprecedented possibility to evaluate the therapeutic effect of drugs on vascular fatty lesions in vivo. To demonstrate this approach, we tested two drugs (atorvastatin and ezetimibe) that have been commonly prescribed to treat patients of hyperlipidemia. These two drugs have demonstrated effects in lowering lipids in plasma but through distinct mechanisms. We describe the results in subsequent sections.

Both Atorvastatin and Ezetimibe Suppressed the Vascular Accumulation of Lipids. We divided fish into three groups according to the protocol displayed (Figure 3A). The intervened groups comprised zebrafish fed with HCD for $5 \mathrm{~d}$ followed with a mixture of HCD and a drug of a designated dose for an additional $5 \mathrm{~d}$; the control comprised zebrafish fed with $\mathrm{HCD}$ alone for $10 \mathrm{~d}$. For comparison, the result obtained 


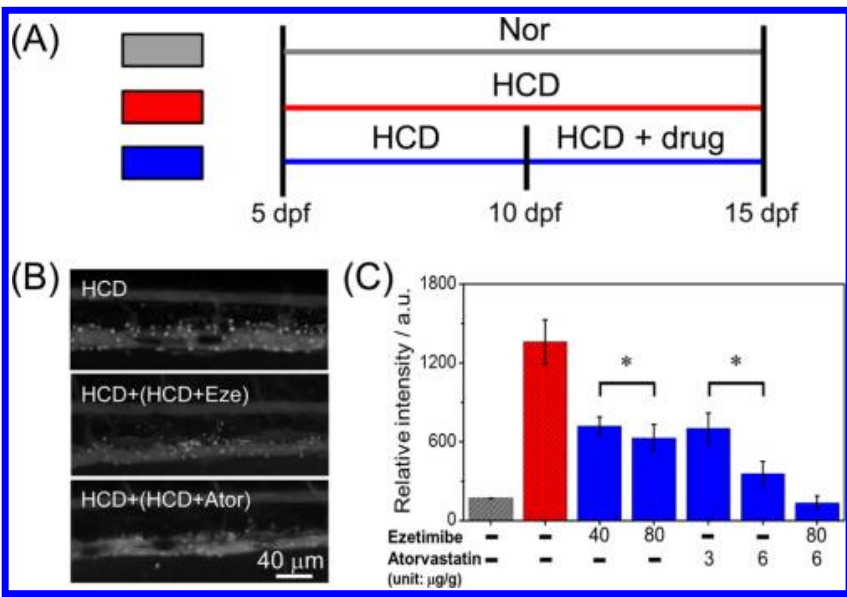

Figure 3. Quantification of the therapeutic effect of drugs on suppressing the accumulation of vascular lipids. (A) Experimental protocol. (B) Representative images of the blood vessels of zebrafish fed with HCD (denoted as "HCD"), with HCD followed by a cotreatment with ezetimibe $(80 \mu \mathrm{g} / \mathrm{g}$; "HCD+(HCD+Eze)"), and with HCD followed by a cotreatment with atorvastatin $(6 \mu \mathrm{g} / \mathrm{g}$; "HCD +(HCD+Ator)"). (C) Quantification of the vascular lipids of zebrafish that were intervened with drugs of indicated doses. The bars with slash lines are results obtained from the baseline and the control that have been shown in Figure 2B.

on zebrafish that were fed with normal diets for $10 \mathrm{~d}$ was included.

As shown from the images, both ezetimibe $(80 \mu \mathrm{g} / \mathrm{g})$ and atorvastatin $(6 \mu \mathrm{g} / \mathrm{g})$ effectively inhibited the accumulation of lipids in the vascular wall (Figure 3B). The efficacy increased with the dose $(48 \%$ and $61 \%$ decrease relative to the control for 40 and $80 \mu \mathrm{g} / \mathrm{g}$ of ezetimibe, and $52 \%$ and $84 \%$ decrease for 3 and $6 \mu \mathrm{g} / \mathrm{g}$ of atorvastatin); interestingly, a combined dose of the two drugs exhibited a synergistic effect in decreasing the deposition of lipids in the vascular wall to nearly the baseline (Figure 3C).
These results have implications. As described, there is extensive clinical evidence that both atorvastatin and ezetimibe are effective in decreasing LDL in the plasma of patients with hyperlipidemia. ${ }^{29,30}$ As the vascular fatty lesions are formed through the entry and accumulation of LDL in the vascular wall, a significantly decreased deposition of lipids in the vascular wall of zebrafish after treatment with either drug is hence consistent with the known therapeutic activity of these two drugs to human beings.

Only Atorvastatin, But Not Ezetimibe, Exhibited an Antioxidative Capability for the Vascular Lipids. As described, LDL that constitutes the main fatty component of atherosclerotic lesions is vulnerable to oxidation. To serve as benchmarks of the oxidative status of vascular fatty lesions, we characterized both native and oxidatively modified LDL; the latter was prepared on incubation with $10 \mu \mathrm{M} \mathrm{CuSO}_{4}$ for $24 \mathrm{~h}$.

As expected, the Raman spectra of LDL exhibited the spectral characteristics of cholesterol (700, 1130, and 1670 $\mathrm{cm}^{-1}$ ) regardless of oxidative modification (Figure 4A). The nonoxidized LDL exhibited two distinctive intense spectral lines at 1155 and $1520 \mathrm{~cm}^{-1}$ (gray bars; upper panel, Figure $4 \mathrm{~A})$; these two lines conform to the $=\mathrm{C}-\mathrm{C}=$ and $-\mathrm{C}=\mathrm{C}-$ stretching modes of the conjugated double bond of $\beta$-carotene, which is an abundant antioxidant in LDL. ${ }^{31,32}$ Most notably, these two lines diminished almost completely after oxidation (lower panel of Figure 4A). The profound sensitivity of these two Raman lines to oxidation indicates a possibility to employ these two spectral lines serving as the signature of the oxidative status of vascular lipids.

We proceeded to compare the antioxidative capability of these two drugs. We measured the Raman spectra of individual vascular fatty lesions of zebrafish that were intervened with either ezetimibe $(80 \mu \mathrm{g} / \mathrm{g})$ or atorvastatin $(3 \mu \mathrm{g} / \mathrm{g})$ according to the protocol described before (Figure $3 \mathrm{~A})$. These two doses were chosen as we have shown that the treatment of these two doses possessed comparable efficacies in decreasing the accumulation of vascular lipids (Figure 3C).
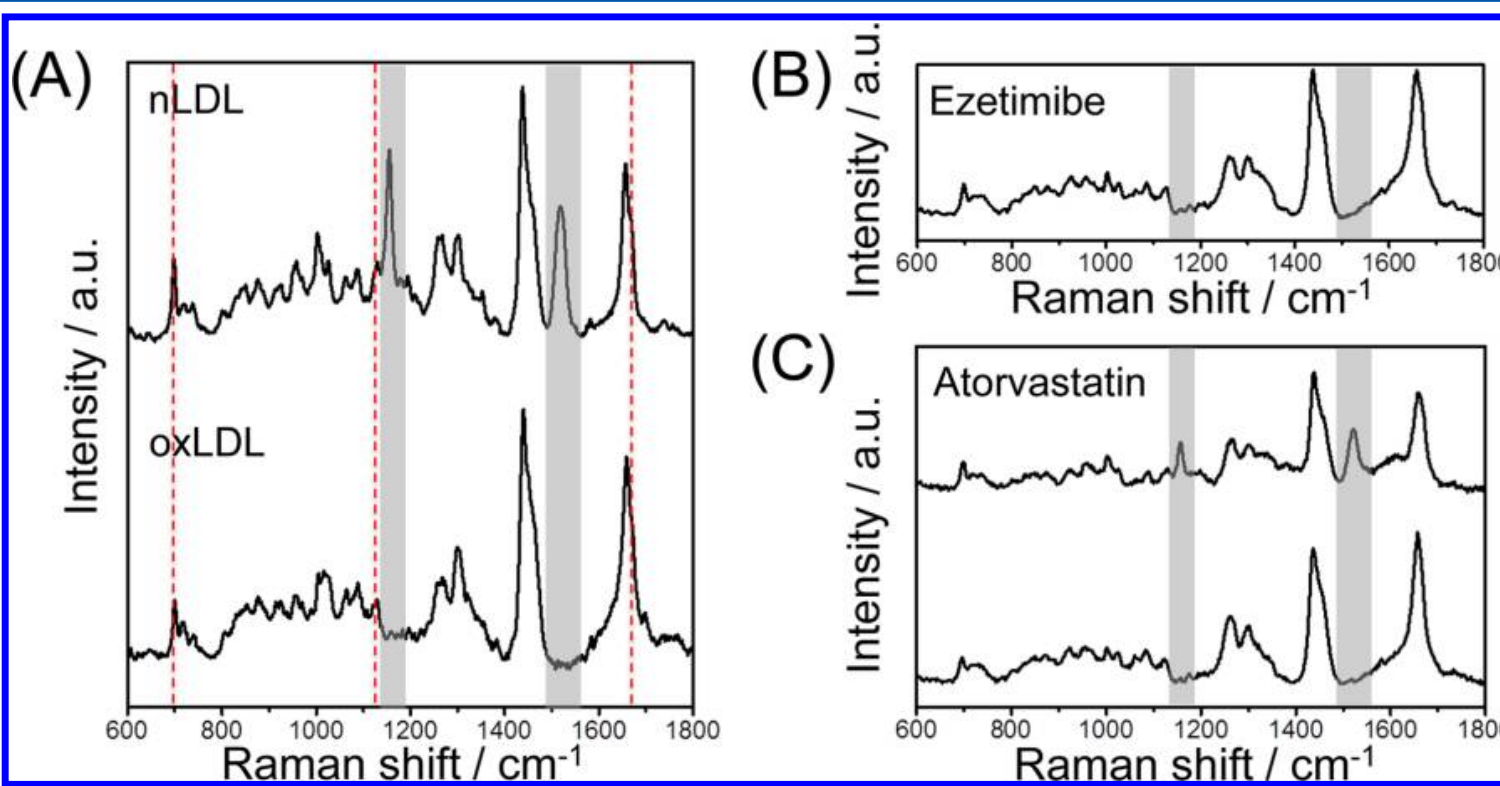

(C)

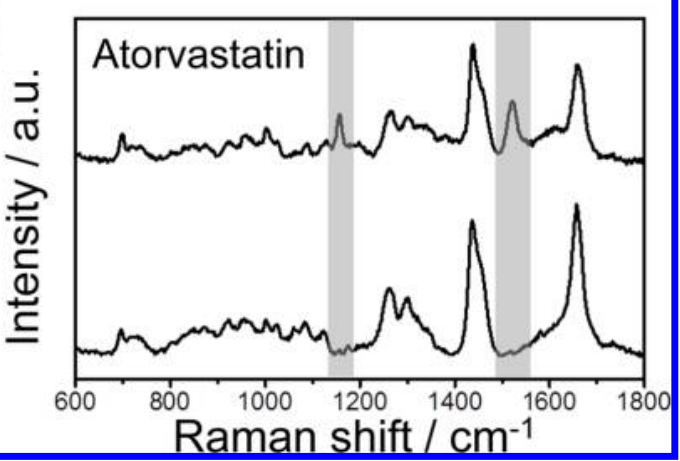

Figure 4. Antioxidative effect of drugs on vascular lipids. (A) Raman spectra of native LDL (nLDL, upper panel) and oxidatively modified LDL (oxLDL, lower panel). (B, C) Representative Raman spectra of vascular fatty lesions measured from fish treated with ezetimibe $(80 \mu \mathrm{g} / \mathrm{g}$; B) and atorvastatin $(3 \mu \mathrm{g} / \mathrm{g} ; \mathrm{C})$. The gray bars and red dashed lines indicate the characteristic Raman lines of $\beta$-carotene and cholesterol, respectively. 


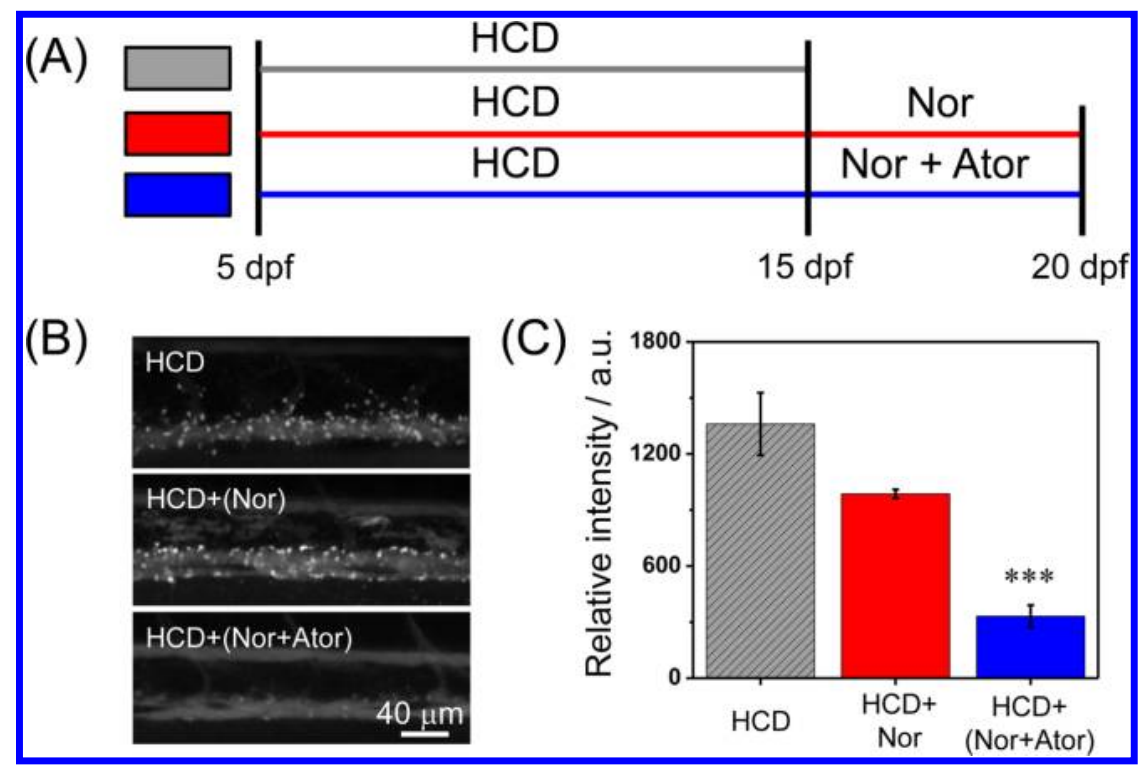

Figure 5. Expedited autoclearance of deposited lipids. (A) Experimental protocol. (B) Representative images of the blood vessels of zebrafish fed with HCD ("HCD"), with HCD followed by normal diets ("HCD+(Nor)"), and with HCD followed by cotreatment with normal diets and atorvastatin $(6 \mu \mathrm{g} / \mathrm{g}$; "HCD+(Nor+Ator)"). (C) Quantification of the vascular lipids. The bar with slash lines is the result obtained from the control ("HCD") that has been shown in Figure 2B.

We measured 12 vascular lesions one by one from six fish treated with ezetimibe. All spectra measured from these vascular lesions consistently exhibited features similar to the spectra of oxidized LDL (Figure 4B vs the lower panel of Figure $4 \mathrm{~A})$; specifically, the two Raman lines $\left(1155\right.$ and $1520 \mathrm{~cm}^{-1}$; gray bars, Figure 4B) associated with $\beta$-carotene diminished almost completely. In striking contrast with the preceding finding, the spectra of individual vascular lesions (19 lesions from six fish) measured after a treatment of atorvastatin comprised two distinct types; among the 19 vascular lesions examined in this test, 4 lesions (upper panel, Figure 4C) exhibited the characteristic features of native LDL (upper panel, Figure 4A), whereas the remaining 15 lesions (lower panel, Figure 4C) conformed to oxidized LDL (lower panel, Figure 4A). Moreover, we found that the ratio of lesions that exhibited Raman characteristics of native LDL increased with the dose of atorvastatin ( 15 out of 36 for a dose of $6 \mu \mathrm{g} / \mathrm{g}$ ).

These results show unambiguously that atorvastatin possessed a distinctive antioxidative capability to vascular fatty lesions, and this antioxidative capability increased with the dose. In contrast, ezetimibe had little effect in suppressing the oxidation of vascular fatty lesions despite its comparable potency in decreasing the deposition of lipids in the vascular wall with that of atorvastatin. In addition to the distinctive therapeutic activities between ezetimibe and atorvastatin, our approach revealed further a striking heterogeneous response of single vascular lesions to the treatment of atorvastatin with respect to antioxidation. This result has mechanistic insight into the activity of atorvastatin on vascular fatty lesions beyond its well-recognized therapeutic effect in lowering the blood lipids, elucidation of which requires further detailed work and will be described elsewhere.

Atorvastatin Expedited the Clearance of Deposited Lipids. Preceding clinical studies using intravascular ultrasound have shown that an aggressive therapy of atorvastatin led to a significant regression in the volume of coronary atherosclerotic lesions, but the detailed mechanism remains elusive. ${ }^{33}$ It is hence interesting to test if atorvastatin exerts a similar beneficial effect to zebrafish by decreasing vascular lipids already deposited in the vascular wall. To address this question, we designed the following protocol (Figure 5A): we examined zebrafish fed with HCD for $10 \mathrm{~d}$ and then with a mixture of a normal diet and atorvastatin $(6 \mu \mathrm{g} / \mathrm{g})$ for an additional $5 \mathrm{~d}$ (denoted as the "HCD+(Nor+Ator)" group); the control corresponded to zebrafish fed with HCD for $10 \mathrm{~d}$ and then with a normal diet for an additional $5 \mathrm{~d}$ (the "HCD+Nor" group). For comparison, the result measured on zebrafish before the replacement of diets was included (the "HCD" group).

As shown from the results (Figures 5B,C), the replacement of HCD with a normal diet ("HCD+Nor") decreased significantly the deposited lipids relative to that measured before the replacement of diets ("HCD"), indicating that autoclearance of the deposited lipids occurred effectively in hypercholesterolemic zebrafish after the replacement of HCD with a normal diet. Furthermore, the administration of atorvastatin together with normal diets ("HCD+(Nor +Ator)") resulted in an additional 55\% decrease in the deposited lipids (Figures 5B,C), indicating that the administration of atorvastatin expedited the clearance of vascular lipids. The finding made on zebrafish is of great significance as it is profoundly consistent with the therapeutic effect of atorvastatin that decreases the volume of atherosclerotic plaques in human beings. ${ }^{33}$ More importantly, the result supports the employment of our approach on zebrafish to screen drugs with prospective atherosclerotic effects.

\section{DISCUSSION}

We have demonstrated for the first time an orchestrated application of confocal fluorescence imaging and Raman spectral measurements to assess the antiatherosclerotic effect of drugs in vivo using the zebrafish model of hypercholesterolemia. Our approach is superior in several respects to conventional means such as biochemical analysis of the lipid profile of plasma or post-mortem histological examination of the blood vessel of a specimen. Specifically, our method enables not only quantification of lipids accumulated in the vascular 
wall but also assessment of their biochemical alteration subject to therapeutic interventions in vivo. Unlike the lipid profile of plasma, the information obtained with our approach is directly related to the pathological abnormalities of vascular walls and is hence of greater clinical relevance. Particularly, our approach enables direct assessment of oxidative modification of the fatty lesions in the vascular wall in situ; this aspect is remarkable given the key role of oxLDL in the pathogenesis of atherosclerosis and vulnerability of plaques. ${ }^{3,5}$ Moreover, guided with fluorescence imaging, we demonstrated an interrogation of vascular fatty lesions one by one with Raman spectral measurements, through which, subtle pharmaceutical effects, such as the heterogeneous response of single vascular fatty lesions to a treatment of atorvastatin, were revealed delicately.

Facilitated with our approach, we show that atorvastatin suppressed effectively the oxidation of vascular lipids and expedited their clearance beyond its potency in decreasing the accumulation of lipids. The possession of pleotropic (multiple) antiatherosclerotic effects by atorvastatin is distinctive and is notably consistent with the pharmaceutical effect of this drug on human beings. This finding has significant implications for pharmacological development as it justifies the utility of our approach to investigate novel interventions targeting atherosclerosis.

\section{CONCLUSION}

We demonstrate a novel means to assess the therapeutic effects of antiatherosclerotic drugs on a zebrafish model of human atherosclerosis in vivo and reveal distinctive pleotropic effects of atorvastatin on the vascular fatty lesions of hypercholesterolemic zebrafish. Our approach is extensible to screen drugs with prospective antiatherosclerotic efficacies and to investigate their therapeutic mechanisms.

\section{AUTHOR INFORMATION}

\section{Corresponding Author}

*E-mail: ianliau@mail.nctu.edu.tw.

\section{Notes}

The authors declare no competing financial interest.

\section{ACKNOWLEDGMENTS}

We thank Professors Yuan-Pern Lee and Yaw-Kuen Li (National Chiao Tung University) for generous support and Professor Yung-Jen Chuang (National Tsing Hua University) for technical advice. We acknowledge Taiwan Zebrafish Core Facility (Grant No.: NSC101-2321-B400-014) as the source of zebrafish. National Chiao Tung University, National Science Council, and the MOE-ATU program of Taiwan supported this research.

\section{REFERENCES}

(1) Ross, R. Nature 1993, 362, 801-809.

(2) Lusis, A. I. Nature 2000, 407, 233-241.

(3) Sparrow, C. P.; Doebber, T. W.; Olszewski, J.; Wu, M. S.; Ventre, J.; Stevens, K. A.; Chao, Y. S. I. Clin. Invest. 1992, 89, 1885-1891.

(4) Thomas, A.; Deglon, J.; Lenglet, S.; Mach, F.; Mangin, P.; Wolfender, J. L.; Steffens, S.; Staub, C. Anal. Chem. 2010, 82, 66876694.

(5) Toshima, S.; Hasegawa, A.; Kurabayashi, M.; Itabe, H.; Takano, T.; Sugano, J.; Shimamura, K.; Kimura, J.; Michishita, I.; Suzuki, T.; Nagai, R. Arterioscler., Thromb., Vasc. Biol. 2000, 20, 2243-2247.
(6) Diaz, M. N.; Frei, B.; Vita, J. A.; Keaney, J. F., Jr. N. Engl. J. Med. 1997, 337, 408-416.

(7) Stoletov, K.; Fang, L.; Choi, S. H.; Hartvigsen, K.; Hansen, L. F.; Hall, C.; Pattison, J.; Juliano, J.; Miller, E. R.; Almazan, F.; Crosier, P.; Witztum, J. L.; Klemke, R. L.; Miller, Y. I. Circ. Res. 2009, 104, 952960.

(8) Fang, L. H.; Green, S. R.; Baek, J. S.; Lee, S. H.; Ellett, F.; Deer, E.; Lieschke, G. J.; Witztum, J. L.; Tsimikas, S.; Miller, Y. I. J. Clin. Invest. 2011, 121, 4861-4869.

(9) Fang, L. H.; Miller, Y. I. Free Radical Biol. Med. 2012, 53, 14111420.

(10) Richards-Kortum, R.; Sevick-Muraca, E. Annu. Rev. Phys. Chem. 1996, 47, 555-606.

(11) Stone, N.; Kendall, C.; Shepherd, N.; Crow, P.; Barr, H. J. Raman Spectrosc. 2002, 33, 564-573.

(12) Huang, Y. S.; Karashima, T.; Yamamoto, M.; Hamaguchi, H. Biochemistry 2005, 44, 10009-10019.

(13) Li, M. D.; Cui, Y.; Gao, M. X.; Luo, J.; Ren, B.; Tian, Z. Q. Anal. Chem. 2008, 80, 5118-5125.

(14) Buschman, H. P.; Marple, E. T.; Wach, M. L.; Bennett, B.; Schut, T. C. B.; Bruining, H. A.; Bruschke, A. V.; van der Laarse, A.; Puppels, G. I. Anal. Chem. 2000, 72, 3771-3775.

(15) Petry, R.; Schmitt, M.; Popp, J. ChemPhysChem 2003, 4, 14-30.

(16) Deng, J. L.; Wei, Q.; Zhang, M. H.; Wang, Y. Z.; Li, Y. Q. J. Raman Spectrosc. 2005, 36, 257-261.

(17) Chan, J. W.; Taylor, D. S.; Lane, S. M.; Zwerdling, T.; Tuscano, J.; Huser, T. Anal. Chem. 2008, 80, 2180-2187.

(18) Majzner, K.; Kaczor, A.; Kachamakova-Trojanowska, N.; Fedorowicz, A.; Chlopicki, S.; Baranska, M. Analyst 2013, 138, 603610.

(19) Chang, W. T.; Lin, H. L.; Chen, H. C.; Wu, Y. M.; Chen, W. J.; Lee, Y. T.; Liau, I. I. Raman Spectrosc. 2009, 40, 1194-1199.

(20) Chang, W. T.; Yang, Y. C.; Lu, H. H.; Li, I. L.; Liau, I. J. Am. Chem. Soc. 2010, 132, 1744-1745.

(21) Ando, J.; Fujita, K.; Smith, N. I.; Kawata, S. Nano Lett. 2011, 11, 5344-5348.

(22) Okada, M.; Smith, N. I.; Palonpon, A. F.; Endo, H.; Kawata, S.; Sodeoka, M.; Fujita, K. Proc. Natl. Acad. Sci. U.S.A. 2012, 109, 28-32.

(23) Huang, C. K.; Ando, M.; Hamaguchi, H.; Shigeto, S. Anal. Chem. 2012, 84, 5661-5668.

(24) Huang, S. H.; Hsiao, C. D.; Lin, D. S.; Chow, C. Y.; Chang, C. J.; Liau, I. PLoS One 2011, 6, No. e24764.

(25) Huang, W. C.; Hsieh, Y. S.; Chen, I. H.; Wang, C. H.; Chang, H. W.; Yang, C. C.; Ku, T. H.; Yeh, S. R.; Chuang, Y. J. Zebrafish 2010, 7, 297-304.

(26) Inya-Agha, O.; Klauke, N.; Davies, T.; Smith, G.; Cooper, J. M. Anal. Chem. 2007, 79, 4581-4587.

(27) Jhan, J. W.; Chang, W. T.; Chen, H. C.; Lee, Y. T.; Wu, M. F.; Chen, C. H.; Liau, I. Opt. Express 2008, 16, 16431-16441.

(28) Maiti, N. C.; Apetri, M. M.; Zagorski, M. G.; Carey, P. R.; Anderson, V. E. I. Am. Chem. Soc. 2004, 126, 2399-2408.

(29) Nawrocki, J. W.; Weiss, S. R.; Davidson, M. H.; Sprecher, D. L.; Schwartz, S. L.; Lupien, P. J.; Jones, P. H.; Haber, H. E.; Black, D. M. Arterioscler., Thromb., Vasc. Biol. 1995, 15, 678-682.

(30) Tremblay, A. J.; Lamarche, B.; Cohn, J. S.; Hogue, J. C.; Couture, P. Arterioscler., Thromb., Vasc. Biol. 2006, 26, 1101-1106.

(31) Parker, S. F.; Tavender, S. M.; Dixon, N. M.; Herman, H. K.; Williams, K. P. J.; Maddams, W. F. Appl. Spectrosc. 1999, 53, 86-91.

(32) Kaczor, A.; Baranska, M. Anal. Chem. 2011, 83, 7763-7770.

(33) Nissen, S. E.; Nicholls, S. J.; Sipahi, I.; Libby, P.; Raichlen, J. S.; Ballantyne, C. M.; Davignon, J.; Erbel, R.; Fruchart, J. C.; Tardif, J. C.; Schoenhagen, P.; Crowe, T.; Cain, V.; Wolski, K.; Goormastic, M.; Tuzcu, E. M. JAMA, I. Am. Med. Assoc. 2006, 295, 1556-1565. 